\title{
Turbulent Rayleigh-Bénard convection with polymers: Understanding how heat flux is modified
}

\author{
Roberto Benzi, ${ }^{1}$ Emily S. C. Ching, ${ }^{2,3}$ and Elisabetta De Angelis ${ }^{4}$ \\ ${ }^{1}$ Dipartimento di Fisica, Università di Roma Tor Vergata and INFN, via della Ricerca Scientifica 1, 00133 Roma, Italy \\ ${ }^{2}$ Department of Physics, The Chinese University of Hong Kong, Shatin, Hong Kong \\ ${ }^{3}$ Institute of Theoretical Physics, The Chinese University of Hong Kong, Shatin, Hong Kong \\ ${ }^{4}$ School of Engineering, Cardiff University, Queen's Buildings, The Parade, Cardiff, CF24 3AA, United Kingdom
}

(Received 10 October 2016; published 21 December 2016)

\begin{abstract}
We study how polymers affect the heat flux in turbulent Rayleigh-Bénard convection at moderate Rayleigh numbers using direct numerical simulations with polymers of different relaxation times. We find that heat flux is enhanced by polymers and the amount of heat enhancement first increases and then decreases with the Weissenberg number, which is the ratio of the polymer relaxation time to the typical time scale of the flow. We show that this nonmonotonic behavior of the heat flux enhancement is the combined effect of the decrease in the viscous energy dissipation rate due to the viscosity of the Newtonian fluid and the increase in the energy dissipation rate due to polymers when Weissenberg number is increased. We explain why the viscous energy dissipation rate decreases with the Weissenberg number. Then by carrying out a generalized boundary layer analysis supplemented by a space-dependent effective viscosity from the numerical simulations, we provide a theoretical understanding of the change of the heat flux when the viscous energy dissipation rate is held constant. Our analysis thus provides a physical way to understand the numerical results.
\end{abstract}

DOI: 10.1103/PhysRevE.94.063110

\section{INTRODUCTION}

It is well known that adding polymers to turbulent wallbounded flows can significantly reduce the friction drag or, equivalently, increase the mass transport. The phenomenon of turbulent drag reduction by polymers has been extensively studied since its discovery by Toms in 1948 [1]. Compared to drag reduction, the effect of polymers on the heat transport in turbulent thermal convection is much less explored and has been the focus of several recent studies [2-5]. RayleighBénard (RB) convection [6-9] is a paradigm system to study turbulent thermal convection. In the RB system, a fluid is constrained between two horizontal plates that are heated from below and cooled from above, and the system is controlled by two parameters: the Rayleigh number, $\mathrm{Ra}=\alpha g \Delta T H^{3} /\left(\kappa v_{s}\right)$, which measures the thermal forcing due to the temperature difference $\Delta T$ between the two plates, and the Prandtl number, $\operatorname{Pr}=v_{s} / \kappa$, which is the ratio between the kinematic viscosity $\nu_{s}$ and the thermal diffusivity $\kappa$ of the fluid. In addition, $\alpha$ is the isobaric volume expansion coefficient of the fluid, $g$ is the acceleration due to gravity, and $H$ is the vertical distance between the top and bottom plates. In turbulent RB convection, there are distinct flow regions, namely viscous boundary layers near all rigid walls and two thermal boundary layers, one above the bottom plate and one below the top plate, and an approximately homogeneous bulk flow in the central region of the convection cell.

The bulk flow of turbulent RB convection without the boundary layers is believed [10] to be a good approximation of the ultimate regime [11] at large $\mathrm{Ra}$. To study the effect of polymers in this case, we have performed direct numerical simulations (DNS) of homogeneous turbulent thermal convection without boundaries using periodic boundary conditions [2] and found that polymers enhance heat transport. A similar heat enhancement by polymers has been found in Rayleigh-Taylor turbulence [4]. At variance with these results, experiments on turbulent RB convection of water at moderate $\mathrm{Ra}$ [3] reveal that polymers reduce heat transport and the amount of reduction increases with polymer concentration. This reduction of heat transport by polymers at moderate $\mathrm{Ra}$ has also been found in a later experimental study [5]. To account for these experimental findings, some of us [12] have generalized the classical boundary layer theory [13-15] to study heat transport by boundary layer flow with polymers using the Oldroyd-B model [16], in which polymer chains can be stretched indefinitely without a limit. We recall that the classical boundary layer theory was developed for steadystate forced convection above a semi-infinite weakly heated plate $[17,18]$ and has been shown $[7,19]$ to provide basic understanding of the physics of heat transport in fluctuating boundary layers in turbulent RB convection at moderate $\mathrm{Ra}$. On the other hand, experiments using convection cell with rough top and bottom plates indicated that heat flux is enhanced by polymers when the concentration of polymers is not too small $[15,20]$. It is believed that the pyramidal structures of the rough plates perturb the boundary layers and make the flow resembling that of the bulk flow even at moderate Ra.

Our work in Ref. [12] and its extension to finitely extensible polymers [21] shows that polymer stretching gives rise to a space-dependent effective viscosity in boundary layer flow. For the Oldroyd-B polymers and for polymers with large finite extensibility, the effective viscosity vanishes quickly as one moves away from the plate and is nonzero only within a region very close to the plate. In both cases, a reduction in heat transport is found and the amount of heat reduction increases with polymer concentration, in agreement with the experimental observations in Ref. [3]. It is thus reasonable to assume that the specific details of polymer stretching determine heat transport in the system.

In this paper, we investigate turbulent $\mathrm{RB}$ convection at moderate Ra with Oldroyd-B polymers of different relaxation times and thus different stretching behaviors. The remainder of this paper is organized as follows. In Sec. II we give a brief review of our earlier DNS study of the bulk flow without boundary layers [2] and the generalization of the classical boundary 
layer theory in the presence of polymers [12]. In Sec. III we report the results of our present DNS of turbulent RB convection at moderate Ra with polymers of different relaxation times and discuss how we can use the generalized boundary layer analysis to provide a theoretical understanding of the change in heat flux due to polymers. Conclusions follow in Sec. IV.

\section{REVIEW OF PREVIOUS RESULTS}

In the Boussinesq approximation, the equations of motion for RB convection with polymers are

$$
\begin{gathered}
\frac{D u_{a}}{D t}=-\nabla_{a} p+v_{s} \nabla^{2} u_{a}+\nabla_{b} \mathcal{T}_{a b}+\alpha g\left(T-T_{*}\right) \delta_{a z}, \\
\frac{D T}{D t}=\kappa \nabla^{2} T,
\end{gathered}
$$

where $D / D t \equiv \partial_{t}+\vec{u} \cdot \vec{\nabla}$ is the material derivative, $\vec{u}$ is the velocity field with components $u_{a}, a=x, y, z$, with $x$ and $y$ along the horizontal directions and $z$ along the vertical direction, $p$ is the pressure, and $T$ is the temperature field with $T_{*}$ being the mean temperature averaged over time and the whole system. We have used the repeated indices summation convention and for simplicity the density of the fluid is taken to be 1 . The polymeric stress tensor $\mathcal{T}_{a b}$ depends on how much the polymer chains are stretched and is a function of the polymer conformation tensor $R_{a b}$. Let $\vec{d}$ be the end-to-end distance vector of a polymer chain, and $R_{a b}$ is the ensemble average of $d_{a} d_{b}$ normalized by the equilibrium value of the ensemble average of $d_{a}^{2} / 3$. For Oldroyd-B polymers,

$$
\mathcal{T}_{a b}(\vec{r}, t)=\frac{v_{p}}{\tau}\left[R_{a b}(\vec{r}, t)-\delta_{a b}\right]
$$

and the equation of motion for $R_{a b}$ is

$$
\frac{D R_{a b}}{D t}=\partial_{c} u_{a} R_{c b}+R_{a c} \partial_{c} u_{b}-\frac{1}{\tau}\left(R_{a b}-\delta_{a b}\right) .
$$

Here $v_{p}$ is the polymer contribution to the zero-shear viscosity of the polymer solution, which increases with polymer concentration, and $\tau$ is the relaxation time of the polymers. Heat transport is measured by the dimensionless Nusselt number $(\mathrm{Nu})$, which is the normalized heat flux defined by

$$
\mathrm{Nu} \equiv \frac{\left\langle u_{z} T-\kappa \partial_{z} T\right\rangle_{A}}{\kappa \Delta T / H},
$$

where $\langle\cdots\rangle_{A}$ denotes an average over a horizontal plane of the convection cell and time.

Equations (1)-(4) have been studied in two different limits, namely (a) large Ra with periodic boundary conditions [2,4] and (b) moderate Ra with no-slip boundary condition for velocity and isothermal boundary condition for temperature [12]. Case (a) corresponds to the ultimate regime [11] in which $\mathrm{Nu}$ scales as $\mathrm{Ra}^{1 / 2}$. In this case, numerical simulations show an increase of $\mathrm{Nu}$ when polymers are added to the system. For case (b), the effect of polymers has been analyzed in the framework of the classical boundary layer theory, which has been known $[7,19]$ to be a good approximation for moderate $\mathrm{Ra}$ and stable boundary layers. We will review the two cases separately in order to highlight the different physics.

In the ultimate regime, most of the energy dissipation is supposed to occur in the bulk of the system. In this case, polymers enhance the length scale $l_{T}$ of thermal plumes and the correlation of vertical velocity and temperature fluctuations, and $\mathrm{Nu}$ increases with the polymer relaxation time $\tau$ or equivalently, the Weissenberg number Wi, defined by

$$
\mathrm{Wi} \equiv \frac{\tau U_{c}}{H},
$$

where $U_{c}=\sqrt{\alpha g \Delta T H}$. More precisely, we find that $\mathrm{Nu}$ $\sim l_{T}^{3 / 2} \sim \mathrm{Wi}^{3 / 2}$.

On the other hand, the experimental investigation of the problem at moderate $\mathrm{Ra}[3,5]$ reported a small but clear reduction of $\mathrm{Nu}$ in the presence of polymers. At low Ra, most of the energy dissipation is concentrated in the boundary layers, and therefore the numerical results reviewed above for the ultimate regime do not apply. In an attempt to understand these experimental results, some of us have generalized the classical theory for boundary-layer flow with polymers in Ref. [12]. The Prandtl-Blasius $[13,14]$ boundary-layer equation of motion for the steady-state velocity field $u_{x} \hat{x}+u_{z} \hat{z}$ is

$$
u_{x} \partial_{x} u_{x}+u_{z} \partial_{z} u_{x}=v_{s} \partial_{z z}^{2} u_{x}
$$

Here $x$ denotes the direction along the plate, $z$ is the direction perpendicular to the plate, and $u_{x} \rightarrow U$ as $z \rightarrow \infty$. The mainstream velocity $U$ plays the role of the large-scale mean flow velocity in turbulent $\mathrm{RB}$ convection. The crucial point about Eq. (7) is that the viscous term is balanced against the nonlinear advection term and that $\partial_{z} \gg \partial_{x}$. The latter is satisfied for flows with large Reynolds number. Introducing the stream function

$$
\Psi(x, z) \equiv \sqrt{v_{s} x U} \phi(\xi)
$$

with

$$
\xi \equiv \sqrt{\frac{U}{v_{s} x}} z
$$

such that $u_{x}=\partial_{z} \Psi$ and $u_{z}=-\partial_{x} \Psi$, we obtain the famous Blasius equation [14]

$$
2 \phi_{\xi \xi \xi}+\phi \phi_{\xi \xi}=0 \text {. }
$$

Here $\phi_{\xi}$ denotes $\partial_{\xi} \phi$. The no-slip boundary conditions, $u_{x}=$ $u_{z}=0$ at the plate, and the condition $u_{x} \rightarrow U$ far away from the plate lead to

$$
\phi(0)=\phi_{\xi}(0)=0 ; \quad \phi_{\xi}(\infty)=1 .
$$

Following the works of Prandtl [13] and Blasius [14], Pohlhausen [15] obtained also the equation for temperature field. By writing the temperature field as

$$
T(x, z)=T_{0}+\left(T_{1}-T_{0}\right) \theta(\xi),
$$

where $T_{1}$ and $T_{0}$ are respectively the temperatures at the plate and far away from the plate (or at the center of the convection cell in turbulent $\mathrm{RB}$ convection), $\theta(\xi)$ satisfies the equation

$$
2 \theta_{\xi \xi}+\operatorname{Pr} \phi \theta_{\xi}=0
$$

with the boundary conditions

$$
\theta(0)=1 ; \quad \theta(\infty)=0 .
$$


In the presence of polymers described by the Oldroyd-B model, Eq. (7) is modified to [12,21]

$$
u_{x} \partial_{x} u_{x}+u_{z} \partial_{z} u_{x}=v_{s} \partial_{z z}^{2} u_{x}+\frac{v_{p}}{\tau} \partial_{z}\left(R_{x z}\right)
$$

Replacing $v_{s}$ by the zero-shear viscosity $v_{0}=v_{p}+v_{s}$ of the polymer solution in Eqs. (8) and (9), one obtains

$$
-\frac{1}{2} \phi \phi_{\xi \xi}=(1-\gamma) \phi_{\xi \xi \xi}+\frac{\gamma}{\mathrm{Wi}_{b}{\sqrt{\mathrm{Re}_{b}}}_{b}} \partial_{\xi} R_{x z},
$$

where

$$
\gamma \equiv \frac{v_{p}}{v_{s}+v_{p}}
$$

increases with the polymer concentration, and

$$
\begin{aligned}
& \operatorname{Re}_{b}=\frac{U H}{\nu_{0}}, \\
& \mathrm{Wi}_{b}=\frac{\tau U}{H}
\end{aligned}
$$

are respectively the Reynolds and Weissenberg numbers of the boundary layer flow. To obtain Eq. (16), we have used the approximation of replacing $x$ by the length $H$ of the (large) plate in the final equation, and this approximation is consistent with the boundary layer description, which is meant to be applicable for $x$ being large. The final step to close the problem is to express $R_{x z}$ in terms of $\phi(\xi)$, which can be done numerically [12,21]. By writing $v_{p} R_{x z}(\xi) \equiv \tau v_{\text {eff }}(\xi) \partial_{z} u_{x} \approx$ $\mathrm{Wi}_{b} \sqrt{\mathrm{Re}_{b}} v_{\text {eff }}(\xi) \phi_{\xi \xi}$ again with the approximation of replacing $x$ by $H$ in the last step, Eq. (16) can always be written as

$$
2 \partial_{\xi}\left\{[1+g(\xi)] \phi_{\xi \xi}\right\}+\phi \phi_{\xi \xi}=0,
$$

where

$$
g(\xi) \equiv \frac{\nu_{\text {eff }}(\xi)}{\nu_{0}}-\gamma
$$

with $g(0)=0$ and $g(\xi) \rightarrow 0$ as $\xi \rightarrow \infty$. The form of the temperature equation [Eq. (13)] remains unchanged, with $\mathrm{Pr}$ becoming $v_{0} / \kappa$ and $\phi(\xi)$ governed by Eq. (20) instead of the Blasius equation [Eq. (10)]. The generalization of the classical boundary layer theory to boundary-layer flow with polymers thus shows that the effect of polymer can be represented by a space-dependent effective viscosity $v_{\text {eff }}(\xi)$. The function $g(\xi)$ can be numerically computed and its specific profile depends on the system parameters, namely $\operatorname{Re}_{b}, \operatorname{Pr}, \mathrm{Wi}_{b}$, and $\gamma$. For the parameters range explored in Ref. [12], $g(\xi)$ is peaked close to the boundary, and a decrease in $\mathrm{Nu}$ compared to the value $\mathrm{Nu}_{0}$ for the Newtonian case with $g(\xi)=0$ (which corresponds to a Newtonian fluid with kinematic viscosity $v_{0}$ ) is observed with the amount of heat reduction increasing with $\gamma$ or the polymer concentration, in qualitative agreement with the experimental observation reported in Ref. [3].

The results from the generalized Prandtl-BlasiusPohlhausen (PBP) boundary-layer theory are interesting for two reasons. First, one can understand the role played by the viscous boundary layers which is relevant for experimental results at moderate $\mathrm{Ra}$. Second, we have shown that polymer effects in the boundary layers are equivalent to a spacedependent effective viscosity. In the following, we show that the profile of the space-dependent effective viscosity is one crucial feature for understanding the effect of polymers on heat transport in turbulent RB convection.

\section{NUMERICAL SIMULATIONS}

In this section, we report our present DNS of turbulent $\mathrm{RB}$ convection at moderate $\mathrm{Ra}$ with polymers of different Wi. Specifically, we integrate numerically Eqs. (1)-(4) in a three-dimensional Cartesian domain of size $8 \times 8 \times 1$ along the horizontal $x$ and $y$ directions with periodic boundary conditions and the vertical direction $z$. The discretization method is a pseudospectral one where Chebychev polynomials are used in the $z$ direction with Fourier modes in the $x$ and $y$ directions. Time integration is performed with a fourth-order Runge-Kutta-Crank-Nicholson scheme. Periodic boundary conditions are imposed at the lateral planes whereas fixedtemperature and no-slip boundary conditions are used at the top and bottom plates. The DNS is performed for a Newtonian fluid of $\operatorname{Pr}=7$ with Oldroyd-B polymers of $v_{p}=0.1 v_{s}$ at different values of Wi using $128^{2}$ (horizontal) $\times 129$ (vertical) fully dealiased modes and polynomials, which yield a spatial resolution large enough to resolve the smallest length scale of the problem. In order to accumulate reliable statistics, data over 30 eddy turnover times are collected after the initial transient is neglected. The Rayleigh number for the Newtonian fluid is $\mathrm{Ra}=2.07 \times 10^{5}$ and the Reynolds number based on $U_{c}, H$, and $v_{s}$ is given by $\operatorname{Re}=U_{c} H / v_{s}=\operatorname{Ra}^{1 / 2} \operatorname{Pr}^{-1 / 2}=172$.

We denote the Nusselt number for the Newtonian case without polymers by $\mathrm{Nu}_{s}$. In Fig. 1 , we show $\mathrm{Nu} / \mathrm{Nu}_{s}$ as a function of Wi. For Wi $=2$ we observe very little change in $\mathrm{Nu}$ with $\mathrm{Nu} / \mathrm{Nu}_{s}=1.008 \approx 1$. For larger $\mathrm{Wi}$, heat flux is enhanced and $\mathrm{Nu} / \mathrm{Nu}_{s}>1$. The amount of heat flux enhancement first increases with $\mathrm{Wi}$, reaching a maximum of about $20 \%$ at Wi around 10, and then decreases. The aim of this paper is to attain a theoretical understanding of this nonmonotonic heat flux enhancement by polymers. Following the discussion in Sec. II, we argue that profile of the polymer stretching plays an important role. In Fig. 2, we show the total amount of polymer stretching, measured by $\left\langle R_{i i}\right\rangle_{A}-3$, as a function of the distance $z$ from the bottom plate for the

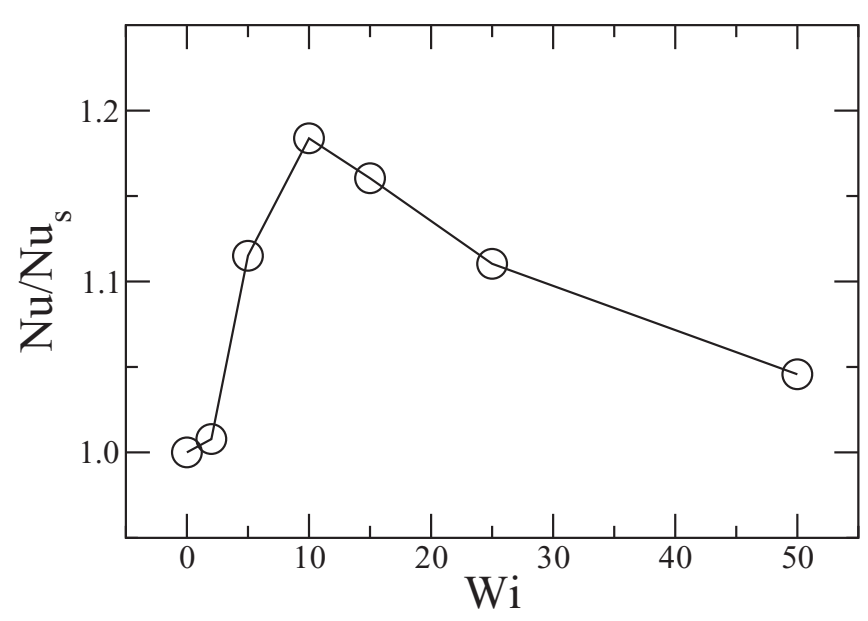

FIG. $1 . \mathrm{Nu} / \mathrm{Nu}_{s}$ as a function of $\mathrm{Wi} . \mathrm{Wi}=0$ corresponds to the Newtonian case without polymers. 


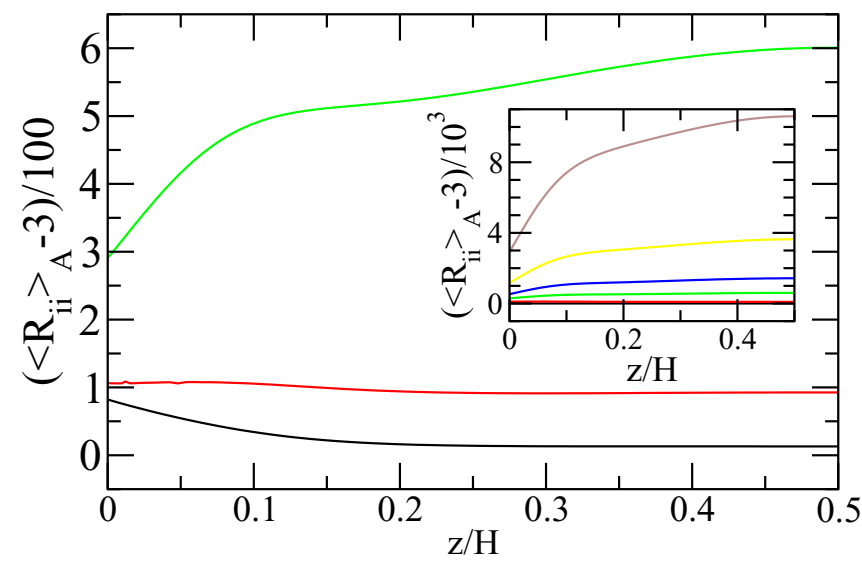

FIG. 2. $\left\langle R_{i i}\right\rangle_{A}-3$ for $\mathrm{Wi}=2,5$, and 10 increasing from the bottom to the top. The results for $\mathrm{Wi}=2$ are multiplied by a factor of 30 for clarity purpose. Inset is the plot for all the values of $\mathrm{Wi}=2$, $5,10,15,25$, and 50 increasing from the bottom to the top.

different values of Wi. For small Wi $(\mathrm{Wi}=2)$, the amount of polymer stretching is negligible and this is consistent with the very slight change in $\mathrm{Nu}$ observed. The amount of polymer stretching increases significantly as Wi is increased. Moreover, we observe the interesting result that polymer stretching is extended into the bulk region of the flow and reaches a maximum at the center of the cell for large $\mathrm{Wi}(\mathrm{Wi} \geqslant 10)$. We will return to this feature below.

It might appear that the monotonic increase of the amount of polymer stretching with $\mathrm{Wi}$ is in contradiction with the observed nonmonotonic heat flux enhancement. We will first understand why this is not so. Taking the dot product of Eq. (1) with $\vec{u}$ and averaging over time and whole volume of the flow, we obtain

$$
\alpha g\left\langle u_{z} T\right\rangle_{V}=\varepsilon_{u}+\varepsilon_{p}
$$

where

$$
\begin{aligned}
\varepsilon_{u} & \equiv v_{s}\left\langle\left(\nabla_{a} u_{a}\right)^{2}\right\rangle_{V}, \\
\varepsilon_{p} & \equiv\left\langle-u_{a} \nabla_{b} T_{a b}\right\rangle_{V},
\end{aligned}
$$

and $\langle\cdots\rangle_{V}$ denotes an average over time and the whole volume of the convection cell. $\varepsilon_{u}$ is the viscous energy dissipation rate due to the viscosity of the Newtonian fluid while $\varepsilon_{p}$ is the rate of energy transfer from the fluid to the polymers which is also equal to the energy dissipation rate due to polymers as the energy transfer to the polymers is eventually dissipated as heat. Using Eq. (5), one can show that

$$
\mathrm{Nu}=\frac{\left\langle u_{z} T\right\rangle_{V}}{\kappa \Delta T / H}+1
$$

Equations (22) and (25) give the exact balance between the heat flux and the sum of the two energy dissipation rates:

$$
\operatorname{RaPr}(\mathrm{Nu}-1)=\frac{H^{4}}{\kappa^{3}}\left(\varepsilon_{u}+\varepsilon_{p}\right) .
$$

Thus, the change in $\mathrm{Nu}$ is the combined effect of the changes in $\varepsilon_{u}$ and $\varepsilon_{p}$ with the latter change being a direct result of polymer stretching. Since the amount of polymer stretching and thus $\varepsilon_{p}$ increases monotonically with $\mathrm{Wi}$ as shown in Fig. 2, the observed nonmonotonic heat flux enhancement must be due to a decrease of $\varepsilon_{u}$ with Wi in some range of Wi. As discussed above, the amount of polymer stretching is negligible at $\mathrm{Wi}=2$; thus we approximate $\varepsilon_{p} \approx 0$ for $\mathrm{Wi}=2$ and denote the value of $\varepsilon_{u}$ at Wi $=2$ by $\varepsilon_{u 0}$. In Fig. 3 we plot $\varepsilon_{u} / \varepsilon_{u 0}$ as a function of $\mathrm{Wi}$, which shows clearly that $\varepsilon_{u}$ indeed decreases with Wi.

In order to understand why $\varepsilon_{u}$ decreases when $\mathrm{Wi}$ is increased, we recall that polymers affect a turbulent flow for scales smaller than or equal to the Lumley scale $l_{L}$, which is defined as the scale at which the time scale of energy transfer given by $l_{L} / \delta v\left(l_{L}\right)$ is equal to the polymer relaxation time. Namely,

$$
\frac{l_{L}}{\delta v\left(l_{L}\right)}=\tau \text {. }
$$

Here $\delta v(r)$ is the characteristic velocity scale of eddies of a characteristic length scale $r$. The effect of polymers is strongest at scales around $l_{L}$. Since $\delta v(r) \sim r^{\beta}$ with an exponent $0<$ $\beta<1, l_{L}$ increases with $\tau$ or Wi. When $l_{L}$ approaches the system size $H$, polymers would interfere with the convective instability that injects energy to the system and leads to a decrease in $\varepsilon_{u}$. This is exactly what happens in our numerical simulations and a similar effect has also been observed for the ultimate regime studied in Ref. [2].

Next, we aim to provide a theoretical explanation of the change in $\mathrm{Nu}$ due to polymers when $\varepsilon_{u}$ is held fixed. Using Eq. (26) with $\mathrm{Nu}-1 \approx \mathrm{Nu}$ we obtain

$$
\frac{\mathrm{Nu}}{\mathrm{Nu}_{0}}=\frac{\varepsilon_{u}}{\varepsilon_{u 0}}+\frac{\varepsilon_{p}}{\varepsilon_{u 0}}
$$

where $\mathrm{Nu}_{0}$ is the value of $\mathrm{Nu}$ at $\mathrm{Wi}=2$ and we have used the approximation that $\varepsilon_{p} \approx 0$ at $\mathrm{Wi}=2$. If $\varepsilon_{u}$ is held fixed at $\varepsilon_{u 0}$, the relative change in heat flux, denoted by $\delta \mathrm{Nu} / \mathrm{Nu}_{0}=\mathrm{Nu} / \mathrm{Nu}_{0}-1$, is given by $\varepsilon_{p} / \varepsilon_{u 0}$. Our basic idea is that the relative change in the heat flux at a fixed viscous energy dissipation rate can be computed using the generalized PBP boundary layer theory, namely

$$
\frac{\mathrm{Nu}}{\mathrm{Nu}_{0}}-\frac{\varepsilon_{u}}{\varepsilon_{u} 0} \equiv\left(\frac{\delta \mathrm{Nu}}{\mathrm{Nu}_{0}}\right)_{\varepsilon_{u} \mathrm{fixed}} \sim\left(\frac{\delta \mathrm{Nu}}{\mathrm{Nu}_{0}}\right)_{\mathrm{PBP}} .
$$

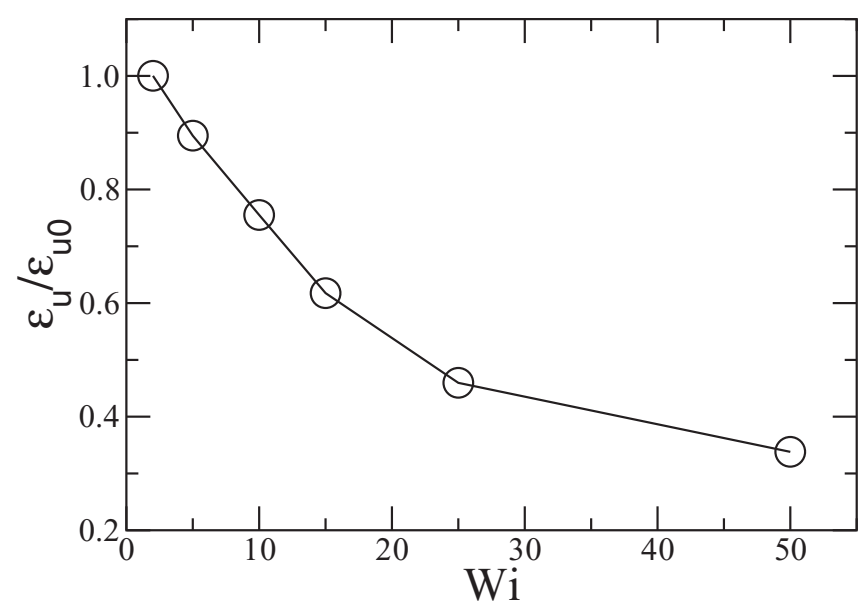

FIG. 3. $\varepsilon_{u} / \varepsilon_{u 0}$ as a function of Wi, where $\varepsilon_{u 0}$ denotes the value of $\varepsilon_{u}$ at $\mathrm{Wi}=2$. 
As discussed above, the polymer stretching extends into the bulk region of the flow when $\mathrm{Wi}>2$ (see Fig. 2). This feature is in contrast to the case in laminar steady-state boundarylayer flow with polymers in which the effective viscosity vanishes quickly as one moves away from the boundary, and is nonzero only within a region very close to the boundary. This indicates that we cannot compute the effective viscosity profile due to polymer stretching self-consistently within the generalized PBP boundary layer theory. Thus we estimate $g(\xi)$ from the DNS data and use it as an external input to the generalized PBP boundary layer theory. We recall that the classical PBP boundary layer theory is in good agreement with both experimental measurements and numerical simulations obtained in turbulent RB system at moderate $\mathrm{Ra}$ and argue that our investigation can provide a way to theoretically understand $\left(\delta \mathrm{Nu} / \mathrm{Nu}_{0}\right)_{\varepsilon_{u} \text { fixed }}$.

To obtain $g(\xi)$ from the DNS data, we note from Eq. (21) that $g(\xi)$ is related to the space-dependent effective viscosity $\nu_{\text {eff }}(\xi)$ of the polymers. We can now use our numerical simulations to get a quantitative computation of $\nu_{\text {eff }}(\xi)$. In our case, the total rate of energy change of the fluid, averaged over time and the horizontal plane of the cell, is the sum of $\varepsilon_{u}(z)=v_{s}\left\langle\left(\nabla_{a} u_{a}\right)^{2}\right\rangle_{A}$ and the rate of energy dissipation due to polymers $E_{p}(z)$. The latter is given in terms of the polymer stretching

$$
E_{p}(z)=\frac{1}{2} \frac{v_{p}}{\tau^{2}}\left[\left\langle R_{i i}\right\rangle_{A}(z)-3\right]
$$

One can define a space-dependent effective viscosity $v_{\text {eff }}(z)$ of polymers by the relation

$$
\frac{v_{\mathrm{eff}}(z)}{v_{s}}=\frac{E_{p}(z)}{\varepsilon_{u}(z)}
$$

and estimate

$$
g(\xi)=\frac{\nu_{\text {eff }}(\xi)-\nu_{\text {eff }}(0)}{\nu_{s}} .
$$

To relate $\xi$ to $z$, we first approximate the case of $\mathrm{Wi}=2$ in the DNS to correspond to a Newtonian fluid with a kinematic viscosity equal to the zero-shear viscosity of the polymer solution $v_{0}=v_{s}+v_{p}$, or equivalently with a Prandtl number $\operatorname{Pr}_{0}=v_{0} / \kappa=7.7$. This is justified by the negligible polymer stretching at $\mathrm{Wi}=2$. Denote the thermal boundary layer thickness by $\lambda_{t 0}$. Then we perform a calculation using the classical boundary layer theory for a Newtonian fluid with $\operatorname{Pr}_{0}=7.7$ and obtain the corresponding $\theta_{0}^{\prime}(0)$ and the (dimensionless) thermal boundary layer thickness $1 /\left|\theta_{0}^{\prime}(0)\right|$. Finally, $\xi$ is related to $z$ by $\xi\left|\theta_{0}^{\prime}(0)\right|=z / \lambda_{t 0}$.

In Fig. 4 we show $g(\xi)$ evaluated from the DNS data as a function of $\xi$. We see the general feature of $g(\xi)$ increases from zero and saturates to some maximum value $A$ near the bulk. We capture this general shape by fitting the data by $g(\xi)$ :

$$
g(\xi)=A\left\{1-\exp \left[-\left(\xi / \xi_{0}\right)^{\alpha}\right]\right\} .
$$

The fitted values of $\xi_{0}$ are around 1 for all $\mathrm{Wi} \geqslant 5$ and the fitted value of $\alpha$ increases from about 1 for $\mathrm{Wi}=5$ to 3 for $\mathrm{Wi}=50$. The idea is to use Eqs. (13) and (20) with this fitted form of $g(\xi)$ [Eq. (33)] to calculate $\left(\delta \mathrm{Nu} / \mathrm{Nu}_{0}\right)_{\mathrm{PBP}}$ as a function of Wi using the generalized PBP theory. As polymer stretching extends to the bulk region and beyond the thermal boundary layers,

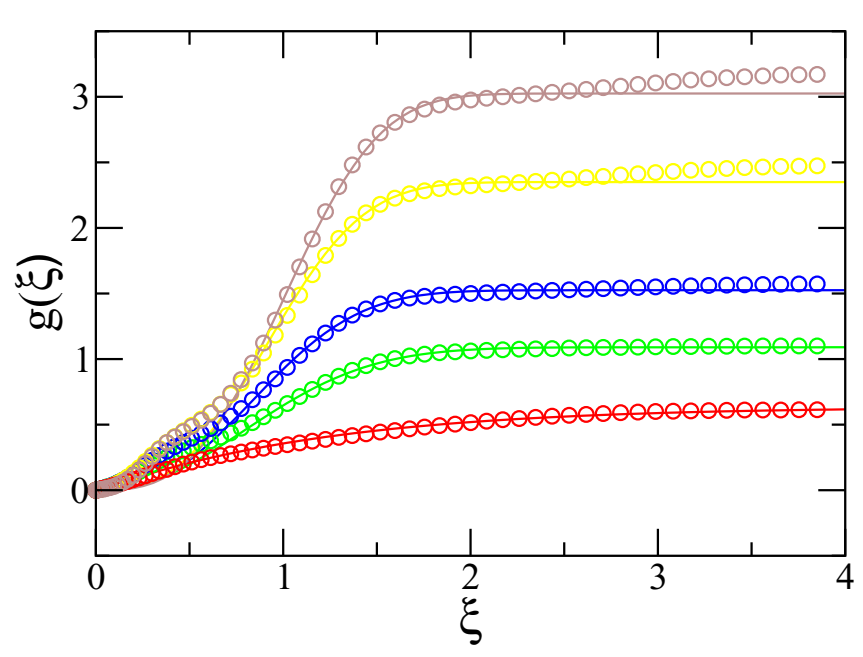

FIG. 4. The function $g(\xi)$, which is evaluated using the DNS data, measures the effective viscosity of the polymers for different Wi as a function of the dimensionless distance $\xi$. Wi $=5,10,15,25$, and 50 from bottom to top. The solid lines are the fits using the simplified form in Eq. (33).

heat flux enhancement is expected [21] and is indeed found. Moreover, we check that $\varepsilon_{u}$ for the boundary layer flow remains approximately constant for all the effective viscosity profiles studied. We now validate the basic assumption in Eq. (29), namely

$$
\left(\frac{\delta \mathrm{Nu}}{\mathrm{Nu}_{0}}\right)_{\varepsilon_{u} \text { fixed }} \sim\left(\frac{\delta \mathrm{Nu}}{\mathrm{Nu}_{0}}\right)_{\mathrm{PBP}},
$$

in Fig. 5 and confirm that $\left(\delta \mathrm{Nu} / \mathrm{Nu}_{0}\right)_{\varepsilon_{u} \text { fixed }}$ is a simple linear function of $\left(\delta \mathrm{Nu} / \mathrm{Nu}_{0}\right)_{\mathrm{PBP}}$. Thus the generalized PBP theory provides a theoretical way to understand the relative change in $\mathrm{Nu}$ due to the polymers when $\varepsilon_{u}$ is held fixed. Specifically, when $\varepsilon_{u}$ is held fixed, heat flux is enhanced by the polymers

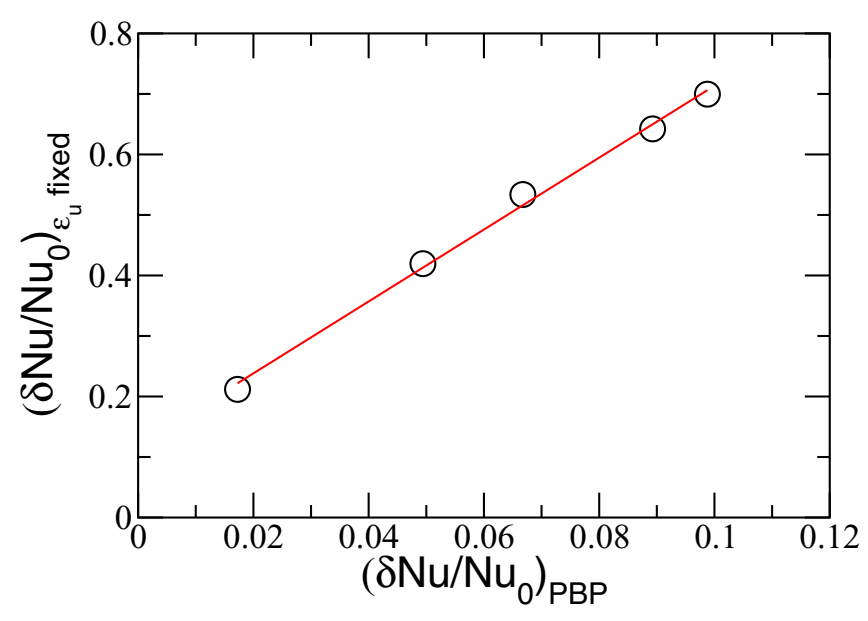

FIG. 5. Comparison of the relative change in $\mathrm{Nu}$ at a fixed energy dissipation rate, $\left(\delta \mathrm{Nu} / \mathrm{Nu}_{0}\right)_{\varepsilon_{u} \text { fixed }}$, measured in the DNS against the theoretical value, $\left(\delta \mathrm{Nu} / \mathrm{Nu}_{0}\right)_{\mathrm{PBP}}$, obtained using the generalized PBP boundary layer analysis with the fitted simplified form of $g(\xi)$ shown in Fig. 4 for $\mathrm{Wi}=5,10,15,25$, and 50. The solid line $y=0.12+$ $5.94 x$ is the least square fit. 


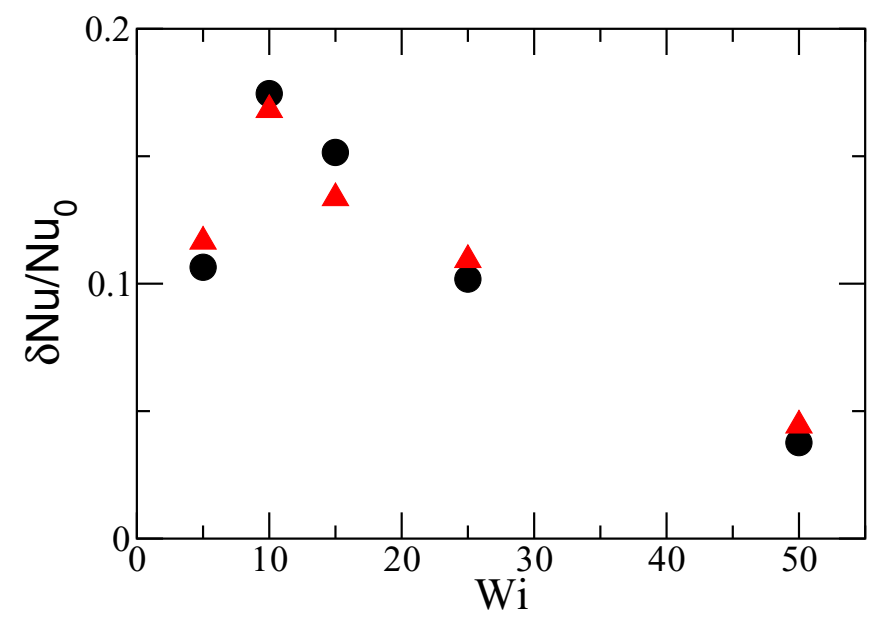

FIG. 6. Comparison of the DNS results of the relative change in $\mathrm{Nu}, \delta \mathrm{Nu} / \mathrm{Nu}_{0}$ (circles), against the results obtained using our present analysis as explained in the text (triangles).

and the relative enhancement in $\mathrm{Nu}$ increases monotonically with Wi as obtained by the generalized PBP theory.

Using the fitted linear relation (see Fig. 5) and the DNS results of $\varepsilon_{u}$ we can obtain $\delta \mathrm{Nu} / \mathrm{Nu}_{0}$ from $\left(\delta \mathrm{Nu} / \mathrm{Nu}_{0}\right)_{\mathrm{PBP}}$ and make a direct comparison of the results so obtained with the DNS results. The good agreement found (see Fig. 6) is another verification of the goodness of the linear fit in Fig. 5.

\section{CONCLUSIONS}

Using DNS we have found that polymers start to affect turbulent RB convection and enhance the heat flux when Wi is greater than 2. For smaller Wi, polymer stretching is negligible. As Wi is increased, the amount of heat flux enhancement first increases but when Wi is beyond around 10, the heat flux enhancement decreases when Wi is further increased. This nonmonotonic behavior of heat flux enhancement is a combined result of decrease in the viscous energy dissipation rate $\varepsilon_{u}$ and the increase in the energy dissipation rate due to polymers $\varepsilon_{p}$ as $\mathrm{Wi}$ is increased. The decrease in $\varepsilon_{u}$ with $\mathrm{Wi}$, which has already been observed in DNS study of the ultimate regime using periodic boundary conditions [2], is due to the increase of the Lumley scale $l_{L}$ with $\mathrm{Wi}$, whereas $\varepsilon_{p}$ increases with $\mathrm{Wi}$ as a result of the increase in polymer stretching. We have analyzed the relative change in heat flux when $\varepsilon_{u}$ is held fixed, $\delta \mathrm{Nu} / \mathrm{Nu}_{\varepsilon_{u}}$ fixed , using the generalized PBP boundary layer theory supplemented by a space-dependent effective viscosity, which represents the effect of polymer stretching. To calculate theoretically the effective viscosity profile, one would need to estimate the amount of polymer stretching in the bulk of the system and its nonlinear coupling with the boundary layers. Such a theoretical development is outside the scope of the present paper. Instead we have used the DNS data to compute the effective viscosity and taken it as an external input for the generalized PBP analysis. Our generalized PBP analysis has demonstrated that heat flux enhancement occurs as polymers are stretched mostly in the bulk of the system and the amount of heat enhancement increases with Wi. We have further confirmed that the values of $\delta \mathrm{Nu} / \mathrm{Nu}_{\varepsilon_{u}}$ fixed are well fitted by a linear function of $\left(\delta \mathrm{Nu} / \mathrm{Nu}_{0}\right)_{\mathrm{PBP}}$ obtained in the generalized PBP analysis. Together with the DNS results of $\varepsilon_{u}$, we are then able to obtain the nonmonotonic heat flux enhancement with Wi. Our analysis thus provides a physical way to understand the DNS results.

Our theoretical approach has the advantage of being quantitative and based on the well-established definition of effective viscosity and momentum balance near the boundary (the Prandtl-Blasius equations). In comparison to the well-known problem of drag reduction by polymers in wall-bounded turbulence, the effect of polymers in turbulent RB convection can be developed using the well-established approach for boundary layer dynamics (with the caveats previously highlighted) and we remark that this is an important point to be exploited in the future. It remains an open question what may happen for large $\mathrm{Ra}$ at which $\mathrm{Nu}$ is dominant from contribution to $\varepsilon_{u}$ from the bulk flow and the physical ground for using the boundary layer approximation breaks down.

\section{ACKNOWLEDGMENTS}

The work of ESCC was supported by the Hong Kong Research Grants Council under Grant No. CUHK-400311.
[1] B. A. Toms, Some observations on the flow of linear polymer solutions through straight tubes at large Reynolds numbers, Proc. 1st Int. Congr. on Rheology 2, 135 (1948).

[2] R. Benzi, E. S. C. Ching, and E. De Angelis, Effect of Polymer Additives on Heat Transport in Turbulent Rayleigh-Bénard Convection, Phys. Rev. Lett. 104, 024502 (2010).

[3] G. Ahlers and A. Nikolaenko, Effect of a Polymer Additive on Heat Transport in Turbulent Rayleigh-Bénard Convection, Phys. Rev. Lett. 104, 034503 (2010).

[4] G. Boffetta, A. Mazzino, S. Musacchio, and L. Vozella, Polymer Heat Transport Enhancement in Thermal Convection: The Case of Rayleigh Taylor Turbulence, Phys. Rev. Lett. 104, 184501 (2010).
[5] P. Wei, R. Ni, and K.-Q. Xia, Enhanced and reduced heat transport in turbulent thermal convection with polymer additives, Phys. Rev. E 86, 016325 (2012).

[6] E. Siggia, High Rayleigh number convection, Annu. Rev. Fluid Mech. 26, 137 (1994).

[7] G. Ahlers, S. Grossmann, and D. Lohse, Heat transfer and large scale dynamics in turbulent Rayleigh-Benard convection, Rev. Mod. Phys. 81, 503 (2009).

[8] D. Lohse and K.-Q. Xia, Small-scale properties of turbulent Rayleigh-Bénard convection, Annu. Rev. Fluid Mech. 42, 335 (2010).

[9] F. Chill'a and J. Schumacher, New perspectives in turbulent Rayleigh-Bénard convection, Eur. Phys. J. E 35, 58 (2012). 
[10] D. Lohse and F. Toschi, Ultimate State of Thermal Convection, Phys. Rev. Lett. 90, 034502 (2003).

[11] R. H. Kraichnan, Turbulent thermal convection at arbitrary Prandtl number, Phys. Fluids 5, 1374 (1962).

[12] R. Benzi, E. S. C. Ching, and V. W. S. Chu, Heat transport by laminar boundary layer flow with polymers, J. Fluid. Mech. 696, 330 (2012).

[13] L. Prandtl, Uber Flüssigkeits bewegung bei sehr kleiner Reibung, Proceedings of the III International Mathematicians Congress (Teubner, Heidelberg, Germany, 1904), pp. 484-491; also available in translation as Motion of fluids with very little viscosity, NACA TM 452, March 1928.

[14] H. Blasius, Grenzschichten in Flüssigkeiten mit kleiner Reibung, Z. Math. Phys. 56, 1 (1908).

[15] E. Pohlhausen, Der Wärmetausch zwischen festen Körpern und Flüssigkeiten mit kleiner Reibung und kleiner Wärmeleitung, Z. Angew. Math. Mech. 1, 115 (1921).
[16] R. B. Bird, O. Hassager, R. C. Armstrong, and C. F. Curtis, Dynamics of Polymeric Liquids (Wiley-Interscience, New York, 1987).

[17] L. D. Landau and E. M. Lifshitz, Fluid Mechanics (Pergamon Press, Oxford, UK, 1987).

[18] H. Schlichting and K. Gersten, Boundary-Layer Theory, 8th ed. (Springer, 2004).

[19] S. Grossmann and D. Lohse, Scaling in thermal convection: A unifying theory, J. Fluid Mech. 407, 27 (2002).

[20] Y.-C. Xie, S.-D. Huang, D. Funfschilling, X.-M. Li, R. Ni, and K.-Q. Xia, Effects of polymer additives in the bulk of turbulent thermal convection, J. Fluid Mech. 784, R3 (2015).

[21] R. Benzi, E. S. C. Ching, W. C. K. Yu, and Y. Wang, Heat transport modification by finitely-extensible polymers in laminar boundary layer flow, J. Fluid Mech. 788, 337 (2016). 